\title{
Two step mechanism for Moreton wave excitations in a blast-wave scenario: the 2006 December 06 case study
}

\author{
G. Krause,,${ }^{1,2 \star}$ M. Cécere,${ }^{2,3 \star}$ C. Francile,${ }^{4}$ A. Costa, ${ }^{1,2,3 \star}$ S. Elaskar ${ }^{1,2}$ \\ and M. Schneiter ${ }^{1,2,3 \dagger}$ \\ ${ }^{1}$ Facultad de Ciencias Exactas, Físicas y Naturales, Universidad Nacional de Córdoba (UNC), Córdoba, Argentina \\ ${ }^{2}$ Consejo Nacional de Investigaciones Científicas y Técnicas (CONICET), Argentina \\ ${ }^{3}$ Instituto de Investigaciones en Astronomía Teórica y Experimental, IATE, Córdoba, Argentina \\ ${ }^{4}$ Observatorio Astronómico Félix Aguilar, Universidad Nacional de San Juan, Argentina
}

Accepted 2015 August 5. Received 2015 August 3; in original form 2015 June 22

\begin{abstract}
We examine the capability of a blast-wave scenario - associated with a coronal flare or to the expansion of CME flanks - to reproduce a chromospheric Moreton phenomenon. We also simulate the Moreton event of 2006 December 06, considering both the corona and the chromosphere. To obtain a sufficiently strong coronal shock - able to generate a detectable chromospheric Moreton wave - a relatively low magnetic field intensity is required, in comparison with the active region values. Employing reasonable coronal constraints, we show that a flare ignited blast-wave or the expansion of the CME flanks emulated as an instantaneous or a temporal piston model, respectively, are capable to reproduce the observations.
\end{abstract}

Key words: MHD - shock waves - Sun: chromosphere-Sun: corona-Sun: flares.

\section{INTRODUCTION}

Moreton waves, a class of large-scale chromospheric disturbances, are detected in emission in the centre and blue wing of the $\mathrm{H} \alpha$ spectral line, whereas they appear in absorption in the red wing, which is interpreted as a compression and subsequent relaxation of the chromosphere (Uchida 1968; Vršnak et al. 2002a). They propagate forming an arc-shaped imprint out of the flare sites, constrained in a certain angular span at distances as long as $500 \mathrm{Mm}$, with radial velocities ranging from 500 to $2000 \mathrm{~km} \mathrm{~s}^{-1}$ (Moreton 1960; Moreton \& Ramsey 1960; Athay \& Moreton 1961).

Although Moreton waves are typically observed in chromospheric spectral lines $(\mathrm{H} \alpha)$, there is consensus that they are of coronal origin since their high speeds are much larger than the characteristic speeds in the chromosphere. Uchida (1968) and Uchida, Altschuler \& Newkirk (1973) proposed a blast-wave scenario where Moreton waves are interpreted as fast-mode magnetohydrodynamic (MHD) shocks expanding in the corona, which produce a chromospheric disturbance due to the shock 'sweeping' over the chromospheric surface. Further reinforcements of this freely propagating large amplitude 'single wave' scenario are the deceleration of the wavefronts, the elongation of the perturbations, and the decreasing amplitude of the disturbances (Warmuth et al. 2001, 2004a). Commonly, the sudden oscillation and winking of distant filaments are

^ E-mail: gustavojavierkrause@gmail.com (GK); cecere@oac.unc.edu.ar (MC); andrea.costa83@gmail.com (AC)

$\dagger$ Present address: Deparment of Astronomy, and Oskar Klein Centre, Stockholm University, Albanova, SE-106 91 Stockholm, Sweden. associated with the passage of the Moreton disturbances (Gilbert et al. 2008; Francile et al. 2013), suggesting that Moreton waves are not always visible at chromospheric levels.

At other wavelengths, similar transient wave-like features were reported, e.g. for the helium I $10830 \AA$ line, soft-X rays and microwaves (Aurass et al. 2002; Vršnak et al. 2002a; Gilbert \& Holzer 2004; Warmuth et al. 2004a; Warmuth, Mann \& Aurass 2005). In the extreme ultraviolet (EUV), global propagating disturbances were first observed by the EUV Imaging Telescope (EIT) aboard the Solar and Heliospheric Observatory ( $\mathrm{SOHO}$; Delaboudinière et al. 1995; Thompson et al. 1998) SOHO and, some authors argue that EIT waves are the coronal counterpart of Moreton waves as they are cospatial (Warmuth et al. 2001, 2004b). Since then, they have been extensively studied giving rise to a controversy regarding the wave or non-wave nature of these 'EIT' (also EUV) disturbances (see White, Balasubramaniam \& Cliver 2014, and references therein). Recent observations give support to the coexistence of a bimodality character of these EIT disturbances: both the wave (fast mode) and the non-wave physical mechanisms can be at work in the same event, although not always detected with the current instrumentation (Zhukov \& Auchère 2004). This would lead to the possibility of multiple driven mechanisms, as proposed by Gilbert \& Holzer (2004) and Zhukov (2011). Also, while some authors find that the EIT and Moreton waves have approximately the same speed, others claim that EIT waves are slower than Moreton waves by a factor of 2-3 (Chen et al. 2002; Chen, Ding \& Fang 2005; Zhang et al. 2011).

Solar flares and coronal mass ejections (CMEs) are atmospheric explosive phenomena capable to produce large-amplitude coronal disturbances and shock waves leading to the formation of a Moreton 
wave. The most straightforward model to give account of the shock formation is a 3D piston mechanism. The flare ignited blast-wave model (or simple 3D shock model) assumes that a temporal piston mechanism is caused by the energy release of a flare-volume expansion that produces an explosion-like process driven by a pressure pulse (Vršnak \& Cliver 2008). On the other hand, the CME-driven shock model proposes that the expansion of a CME, together with the rise of a corresponding flux rope, produces a combination of a piston-shock and a bow-shock, which generates the large-scale shock wave (Chen \& Shibata 2000; Chen et al. 2002). Temmer et al. (2009) stated that the observation of Moreton waves can only be reproduced by applying a strong and impulsive acceleration for the source region expansion acting in a temporal piston mechanism scenario. They proposed that the expansion of the flaring region or the lateral expansion of the CME flanks is more likely the driver of the Moreton wave that the upwards moving CME front.

The flare-CME controversy also extends to the origin of type II radio burst that are usually observed close in time and distance to the shock source. They are interpreted to be the emission, at the local plasma frequency, of shock accelerated electrons producing Langmuir waves (Knock et al. 2001). While there is a consensus that the emission in the decameter or longer wavelength range is associated with CMEs, metric wavelengths can be due either to flare or CME ignited shocks (see e.g. Vršnak \& Cliver 2008). Recent papers show evidence of Type II radio burst associated with a flare without a CME companion (Magdalenić et al. 2012; Su et al. 2015).

Thus, two different views on the origin of large-scale coronal shock waves arise, one favouring CMEs and the other preferring flares. In favour of the flare model it is argued that the required Moreton wave acceleration is larger or more impulsive than the usually observed values for CMEs. However, the large discrepancy between the great number of registered flare events and the relatively rare occurrence of observable coronal shocks (Cliver, Webb \& Howard 1999) suggests that, in addition to the flare explosion, another mechanism could be necessary to produce large-scale waves, or that a very special condition must be accomplished for the shock formation. In fact, following an analysis of orders of magnitude, Vršnak \& Cliver (2008) showed that relatively high values of the plasma parameter (plasma-to-magnetic pressure ratio) $\beta \approx 0.1-0.01$ are required to ignite a coronal shock wave (at least two orders of magnitude larger than in an active region $(\mathrm{AR})$ where $\beta \approx 0.0001$ ). This could explain the rarity of Moreton waves, as they should be triggered at the peripheries of ARs where the magnetic field is decaying. An alternative reasoning of this argument is given in the appendix.

Several numerical simulations have been carried out to try to explain large-scale wave formations in the solar atmosphere. 3D MHD numerical simulations were performed considering a solar flare-induced pressure pulse (Wu et al. 2001), and although the main characteristics of the observed EIT waves were reproduced, the plasma parameter used $\beta \sim 1$, was too large. The CME scenario has also been simulated. In many cases an ad hoc force is used to model the eruptive flux rope that triggers the expansion of the CME flanks and drives the shock (Chen et al. 2002, 2005; Mei et al. 2012). The simulated shock sweeps the chromosphere forming the Moreton wave, where a slower wave (identified as the EIT wave) propagates outwards. Whether the resulting weak coronal CME shock can produce a detectable chromospheric Moreton wave is part of the controversy since to do so the expansion has to be accelerated to velocities which are rare in CMEs.

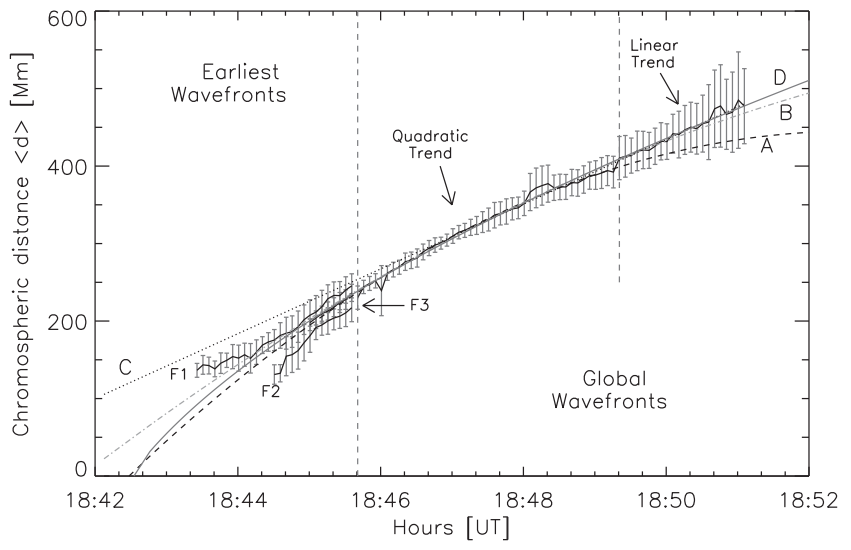

Figure 1. Solid line: 2D averaged chromospheric distance $\langle d\rangle$ from $Q_{0}$ with a $1 \sigma$ dispersion value. Earliest wavefronts $F_{1}, F_{2}, F_{3}$ before $t=18: 45: 40$ ut A: partial quadratic fit. B: Total quadratic fit. C: Partial linear fit. D: Powerlaw fit (taken from Francile et al. 2013).

In Francile et al. (2013) we studied a Moreton wave detected on 2006 December 06, with the $\mathrm{H} \alpha$ Solar Telescope from Argentina (HASTA) in the $\mathrm{H} \alpha$ line $656.3 \mathrm{~nm}$. We determined the kinematics of the whole complex process through a $2 \mathrm{D}$ reconstruction of the HASTA and corresponding TRACE observations (Transition Region And Coronal Explorer; Handy et al. 1999). In Fig. 1 we synthesized the observational results of the chromospheric distances travelled by the Moreton wave as a $2 \mathrm{D}$ planar projection, perpendicular to the line of sight. The evolution of the Moreton wave lead to the activation of two distant filaments. We also noted three initial irregular wavefronts that can be attributed to local inhomogeneities of the coronal medium crossed by the disturbance. We used different fits to describe the kinematics of the event. Curve $D$ is a power-law fit on the complete set of wavefront data. The resulting initial acceleration of the curve is $a_{0} \approx-30.2 \mathrm{~km} \mathrm{~s}^{-2}$ (starting with an initial speed of $s_{0} \geq 2121 \mathrm{~km} \mathrm{~s}^{-1}$ at $t=18: 42: 30 \mathrm{UT}$ ). A partial quadratic fit trend $A$ associated with an acceleration of $a \geq-2.4 \mathrm{~km} \mathrm{~s}^{-2}$ was also used to adjust the data ranging within [18: 45 : 40 - 18: 49: 02] UT with an initial speed of $s_{0} \geq 1463 \mathrm{~km} \mathrm{~s}^{-1}$ (extrapolated at 18: 42: 28 UT). The quadratic acceleration value and the initial speed are higher than those obtained by other authors for the same event (Balasubramaniam et al. 2010). The partial linear fit trend $A$ of the figure corresponds to a free wave speed of $\approx 700 \mathrm{~km} \mathrm{~s}^{-1}$. We concluded that the Moreton wave event observed on 2006 December 6 , can be interpreted as a coronal fast-shock wave of a blast type originated by a single flare source during a CME ejection. However, its onset time is concurrent with the peak of the Lorentz force applied to the photosphere measured by Balasubramaniam et al. (2010) showing an overlap with the flare explosive phase and other minor scale events. This argument favors the hypothesis that the phenomenon can be described as the chromospheric imprint of a fast coronal shock triggered from a single source in association with a CME.

In this work, to reproduce the observational description in Francile et al. (2013), we present a 2D numerical simulation of the 2006 December 6, Moreton wave. The initial configuration is a simplified 2D blast-wave scenario able to trigger a real, freely propagating MHD wave without considering the magnetic field restructuring of a CME. The blast-wave, acting as a piston mechanism is emulated by both, an instantaneous pressure pulse and other one that extends over a short time which could also resemble the action of the flank expansion of a CME. The aim is to discuss whether the 
instantaneous and temporal piston models are capable to reproduce the main characteristics of the observations. We here use the word 'instantaneous' to distinguish a pulse used only as the initial condition, from another one where the pulse is imposed for a given lapse of time of the run, named 'temporal pulse'.

\section{THE MODEL}

The 2D ideal MHD equations for a completely ionized hydrogen plasma, with $\gamma=5 / 3$ ( $\gamma$ the ratio of specific heats) were implemented to study Moreton waves in the frame of the blast-wave scenario. We first use a pressure pulse to simulate the flare-volume expansion (instantaneous piston mechanism) that causes the blastwave propagating fast-mode shock which sweeps the chromosphere (Uchida 1968; Uchida et al. 1973). The pressure pulse emulates the result of different complex processes that can trigger the flare activity and impulsively heat the AR (Wu et al. 2001; Onofri et al. 2004; Guo, Bhattacharjee \& Huang 2013). The ideal MHD equations, in conservative form, result:

$\frac{\partial \rho}{\partial t}+\nabla \cdot(\rho \boldsymbol{v})=0$

$\frac{\partial \rho \boldsymbol{v}}{\partial t}+\nabla \cdot\left(\rho \boldsymbol{v} \boldsymbol{v}-\frac{\boldsymbol{B} \boldsymbol{B}}{4 \pi}+\boldsymbol{I}\left(p+\frac{B^{2}}{8 \pi}\right)\right)=0$

$\frac{\partial \varepsilon}{\partial t}+\nabla \cdot\left(v\left(\varepsilon+p+\frac{B^{2}}{8 \pi}\right)\right)=0$,

where $\rho$ indicates the density, $\boldsymbol{v}$ the velocity, $\boldsymbol{B}$ the magnetic field, $p$ is the pressure and $\varepsilon$ the energy density given by

$\varepsilon=\frac{p}{\gamma-1}+\frac{1}{2} \rho v^{2}$.

As we are interested in low coronal phenomena (flare ignition scenario) and considering typical large values of the coronal pressure scaleheight, $\approx 100 \mathrm{Mm}$, we neglect the gravity term in the equations.

Our aim is to describe the effect of the coronal wave over the transition region and the upper chromosphere. Thus, the chromosphere is modelled as a thin simple layer where the pressure is constant and the temperature and density abruptly change at the transition region. The height of the region is arbitrarily assumed as $5 \mathrm{Mm}$, approximately twice the height of the $\mathrm{H} \alpha$ line core formation (Leenaarts, Carlsson \& Rouppe van der Voort 2012). We assume that the excess of the $\mathrm{H} \alpha$ core emission generated by the compression of the coronal shock is only due to the action of the upper chromosphere (observationally quantified by techniques of running differences). Following Leenaarts et al. (2012) the emission of the $\mathrm{H} \alpha$ line core is strongly correlated with the mass density, and is only weekly modulated by the temperature and the velocity. Thus, the density traces the variations caused by the magnetic field, the waves and shock waves. Also, as stated by Leenaarts et al. (2007) this region can be considered as optically thin outside dynamic magnetic structures as fibrilles.

The shock wave characteristics are given by the coronal properties. Since the ratio of the coronal gas pressure to the magnetic pressure is $\beta \sim c_{\mathrm{s}}^{2} / v_{\mathrm{A}}^{2} \ll 1\left(c_{\mathrm{s}}\right.$ the acoustic speed and $v_{\mathrm{A}}$ the Alfvén speed) the velocity of the coronal fast magnetosonic shock caused by the flare will mainly depend on the magnetic field through the Alfvén speed. Also, as stated by the Rankine-Hugoniot conditions, the observational amplitude of the emitted wave is directly related to the pressure (or density) increase through the shock, i.e. the compression ratio (Vršnak et al. 2002b; Vršnak \& Cliver 2008). The question is, again, if the pressure gradient can produce a sufficiently strong shock wave in the ambient corona able to generate a detectable chromospheric Moreton wave. Although the AR pressure can increase several times according with the admissible values of temperature and density (see e.g. Aschwanden 2005), limitations arise because whereas a sufficiently strong magnetic field is required to reach the correct velocity of the magnetosonic shock, the shock intensity rapidly decays with increasing magnetic fields (see the appendix). Thus, to obtain a sufficiently large perturbation a larger pressure pulse is needed. However, there is an upper limit for the temperature and density values. A typical AR temperature threshold should be as large as $40 \times 10^{6} \mathrm{~K}$, (Aschwanden 2005). An alternative would be to investigate the effect of a temporal pressure pulse, allowing a lower pressure pulse, though acting along time. With the aim of reproducing the observational HASTA results and considering this constraint, we analyse the influence of the magnetic field in the formation of large scale solar waves for a uniform magnetic field assumption.

\subsection{Numerical code and initial conditions}

For the numerical simulations we use the FLASH code developed at the Center for Astrophysical Thermonuclear Flashes (Flash Center) of the University of Chicago (Fryxell et al. 2000). This code, currently in its fourth version, can be used to solve the compressible MHD equations with adaptive mesh refinement (AMR) capabilities. We choose for our simulations the 'Unsplit Staggered Mesh' scheme (Lee, Deane \& Federrath 2009) available in FLASH, which uses a high-resolution finite-volume method with a directionally unsplit data reconstruction and the constraint transport method (CT) to enforce the $\nabla \cdot \boldsymbol{B}=0$ condition. The Riemann problems of the computational interfaces are calculated by a Roe-type solver.

A Cartesian 2D grid with a discretization of $20 \times 10$ cells is used with eight levels of refinement. The refinement criterion takes into account the variations of the density, pressure and magnetic field (the maximum refinement corresponds to a cell of $0.39 \mathrm{Mm}$ ). The physical domain is set to $(1000,500) \mathrm{Mm}$ and consists of two regions that model the solar atmosphere: the chromosphere formed by a small region above the solar surface, and the corona.

Initially the atmosphere is in total equilibrium with a uniform ambient magnetic field (open-field assumption). Thus, the plasma pressure is constant in the initial equilibrium configuration. On the other hand, although in this flare-ignited scenario the interest is focused in describing low coronal features, the physical domain was extended in the vertical direction to avoid possible spurious results generated by the interaction between the shock and the upper boundary. We choose typical values of temperature and number density at the coronal base, $T_{\mathrm{u}}=1.6 \times 10^{6} \mathrm{~K}$ and $n_{\mathrm{u}}=1.2 \times 10^{8} \mathrm{~cm}^{-3}(\mathrm{Wu}$ et al. 2001). The number density in the chromosphere is obtained considering an initial temperature of $T_{\mathrm{d}}=1 \times 10^{4} \mathrm{~K}$, and the same plasma pressure as in the corona. As mentioned, the pressure pulse intensity is limited by the maximum admissible temperature and density, which can increase up to $T \approx 40 \times 10^{6} \mathrm{~K}$ and $n \approx 10^{11} \mathrm{~cm}^{-3}$, e.g. if we consider a flaring loop (Aschwanden 2005).

First, the pressure pulse is instantaneously triggered at $t=0 \mathrm{~s}$. The number density is maintained constant and the temperature is determined by the applied pressure pulse variation, but, if for a given pressure increment the maximum temperature is exceeded, the density is increased to maintain the temperature below the threshold. The size of the pressure pulse is fix to $10 \mathrm{Mm}$, in accordance with typical flare kernel sizes (Vršnak \& Cliver 2008). The distance 


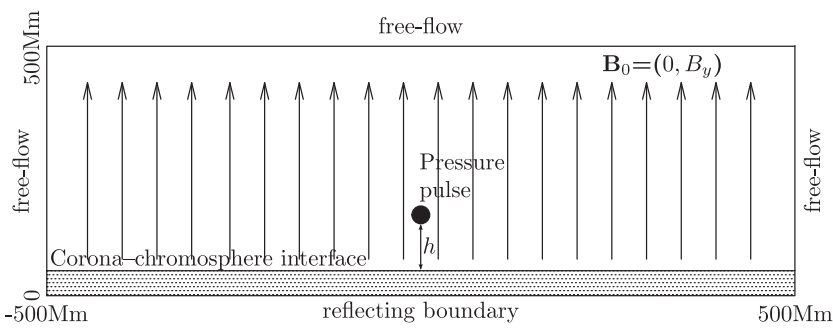

Figure 2. $2 \mathrm{D}$ simulation scheme. Corona: $T_{\mathrm{u}}=1.6 \times 10^{6} \mathrm{~K}$, $n_{\mathrm{u}}=1.2 \times 10^{8} \mathrm{~cm}^{-3}, p_{\mathrm{u}}=0.0265 \mathrm{dyn} \mathrm{cm}^{-2}$. Chromosphere: $T_{\mathrm{d}}=10^{4} \mathrm{~K}$, $p_{\mathrm{d}}=p_{\mathrm{u}}, n_{\mathrm{d}}=1.92 \times 10^{10} \mathrm{~cm}^{-3}$. The chromospheric height is $5 \mathrm{Mm}$ and $h=35 \mathrm{Mm}$ is the distance of the pressure pulse $(\Delta p)$ from the interface corona-chromosphere.

between the pressure pulse location and the chromospheric surface is set to adjust the space-time interval between the flare event and the beginning of the Moreton wave reported by the observations. We assume that the flare occurs near the boundary of the AR where the magnetic field has already decayed and we study the propagation of the perturbation from the boundary of the AR across the quiet corona where the magnetic field is assumed uniform. The magnetic field is varied within $\sim[1-10] \mathrm{G}$ to adjust the phenomenological values of Fig. 1. Secondly, the procedure is repeated using a temporal pressure pulse, i.e. a pulse applied during a lapse of time, to be determined in order to fit the observations. This case could represent the action of the flank expansion of a CME as suggested by Temmer et al. (2009).

Fig. 2 shows the setup of the physical model, with the upper coronal region and the downward chromospheric one. We use freeflow conditions (zero gradient values) for the upper and lateral boundaries and a reflecting condition for the lower boundary to model the denser solar surface values.

\section{RESULTS AND DISCUSSION}

\subsection{Kinematics: a two-step mechanism}

To give account of the two-step mechanism is essential to simulate the chromosphere as a dense thin layer where the coronal perturbation penetrates and rebounds. To understand how these two mechanisms act we analyse Fig. 3. The figure shows a zoom, at $t=300 \mathrm{~s}$, of the numerical simulation domain where a coronal disturbance is triggered by a pressure pulse $\Delta p / p=100$ times larger than the ambient pressure, located at a height $h=35 \mathrm{Mm}$ with a uniform magnetic field $B_{0}=1 \mathrm{G}$. There are two main effects of the shock front over the interface corona-chromosphere: (first step) an intense compression of the chromosphere acting persistently in the vertical direction (note the vertical discontinuity in Figs 3(a)-(d) at $x \sim 18 \mathrm{Mm}$ ), which is first initiated remaining quasi-stationary, and, (second step) a circular shaped shock or chromospheric disturbance, appearing delayed with respect to the initial compression that travels in the corona and 'sweeps' the chromosphere. We note that behind the leading shock wave $(x \sim 120 \mathrm{Mm}$ at a coronal height of $h \sim 35$ $\mathrm{Mm})$ there is a complex pattern of interacting waves. This non-linear interaction is able to weaken the leading shock and consequently the Moreton wave (see a simpler study of the non-linear interaction, where the combined wave front is plane in e.g. Fernández et al. 2009, Costa et al. 2009 and Cécere et al. 2012). Fig. 4 shows a temporal scheme of the second step procedure: the initiation of the travelling chromospheric disturbance. At $\left(x_{C 0}, t_{0}\right)$ the blast coronal wave is triggered. The point $\left(x_{I 1}, t_{1}\right)$ shows the space-time location a) Density: $\rho \times 10^{-15}\left[\mathrm{~cm}^{-3}\right]$

$\begin{array}{lllll}0.08 & 0.5 & 3.2 & 20 & 126\end{array}$
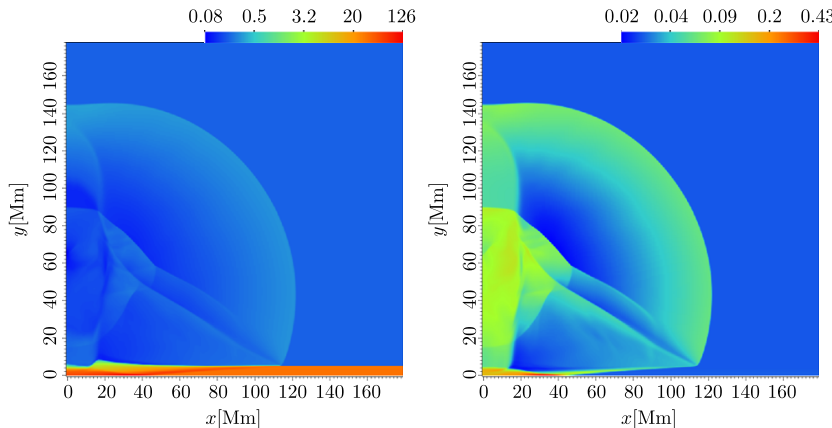

c) Velocity: $v\left[\mathrm{~km} \mathrm{~s}^{-1}\right]$

$\begin{array}{lllll}0 & 59 & 119 & 178 & 238\end{array}$

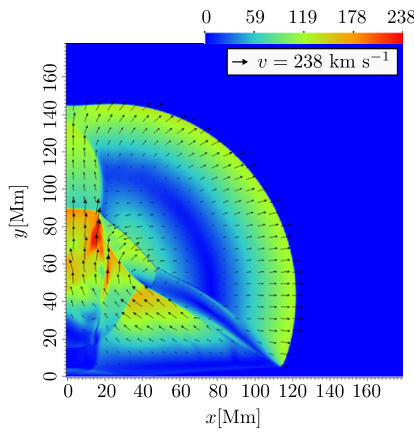

d) Magnetic Field: $B[\mathrm{G}]$

$\begin{array}{lllll}0.16 & 0.51 & 0.86 & 1.20 & 1.55\end{array}$

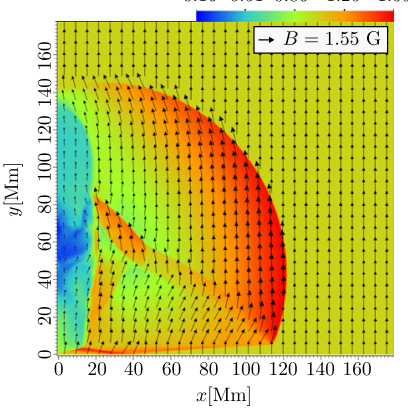

Figure 3. Coronal fast MHD shock at $t=300 \mathrm{~s}$ with $B=1 \mathrm{G}$. Panel (a) shows the density; panel (b) shows the pressure; panel (c) shows the velocity and panel (d) shows the magnetic field.

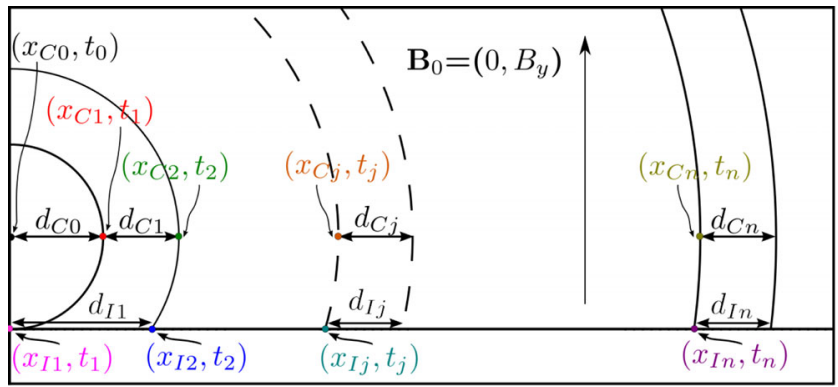

Figure 4. Scheme of the two step mechanism. The flare originates at the corona at $\left(x_{C 0}=0, t_{0}\right)$. The wavefront arrives to the corona-chromosphere interface at $\left(x_{I 1}=0, t_{1}\right)$, when the coronal wavefront has travelled a distance $d_{C 0}$ from the origin. At $t_{2}$, the coronal wavefront has travelled a distance $d_{C 1}$ from $x_{C 1}$ while the chromospheric front has travelled a distance $d_{I 1}>d_{C 1}$. Thus, the chromospheric wavefront speed is larger than the coronal one, $d_{I 1} /\left(t_{2}-t_{1}\right)=\left(x_{I 2}-x_{I 1}\right) /\left(t_{2}-t_{1}\right)>d_{C 1} /\left(t_{2}-t_{1}\right)=\left(x_{C 2}-x_{C 1}\right) /\left(t_{2}\right.$ $\left.-t_{1}\right)$. For later times, the coronal wavefront speed and the chromospheric one become increasingly similar, $d_{I j} /\left(t_{j+1}-t_{j}\right) \simeq d_{C j} /\left(t_{j+1}-t_{j}\right)$. The chromospheric wavefront lags with respect to the coronal one.

where the blast arises to the interface corona-chromosphere (point $\left(x_{C 1}, t_{1}\right)$ is its corresponding coronal counterpart). For each $j,\left(x_{C j}, t_{j}\right)$ and $\left(x_{I j}, t_{j}\right)$ are a coronal and a chromospheric space-time point that belong to the same wavefront. Due to the geometrical characteristic of the scenario, while the wavefront travels from $t_{1}$ to $t_{2}$ a distance $d_{C 1}=x_{C 2}-x_{C 1}$ in the corona, the corresponding intersection, lying in the interface corona-chromosphere, travels a larger distance $d_{I 1}=x_{I 2}-x_{I 1}$. Note that, while for earlier times $d_{C j}<d_{I j}$, for longer ones $d_{C j} \sim d_{I j}$. Therefore, the initial coronal wave speed is slower than the chromospheric one and they become increasingly similar for larger times. This corresponds to a virtual deceleration 


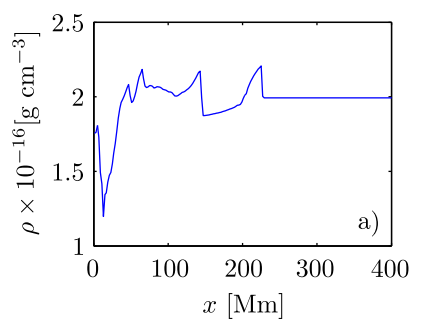

Corona

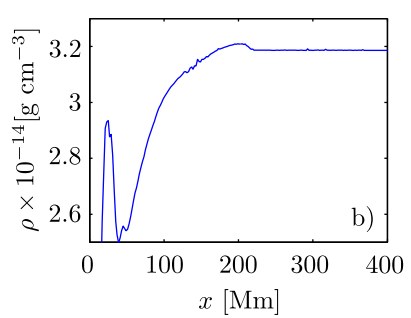

Chromosphere
Figure 5. Density profiles in the solar atmosphere for $t=300 \mathrm{~s}$ and $B_{0}=3 \mathrm{G}, \Delta p / p=100$. (a) corona; (b) chromosphere.

given by the variation of the speed of a virtual point determined by the intersection between the chromosphere and the wavefront. This could give account of the strong initial deceleration of Moreton waves, also explaining why some authors find that these waves are faster than EIT waves (Zhang et al. 2011). Later, when the wavefront is capable to sweep a detectable amount of chromospheric material, a real free wave that lags with respect to its coronal counterpart is observed. In Figs 5(a)-(b) we display two density profile slices: Fig. 5(a) at the coronal level ( $h=35 \mathrm{Mm}$ above the interface corona-chromosphere) and Fig. 5(b) located in the chromospheric upper layer ( $100 \mathrm{~km}$ below the interface corona-chromosphere), at $t=300 \mathrm{~s}$. The initial background magnetic field used is $B_{0}=3 \mathrm{G}$ and the triggering pressure pulse is $\Delta p / p=100$. In the coronal case an evident shock is appreciated (200 Mm $\leq x \leq 230 \mathrm{Mm})$, with a sharp density enhancement followed by a rarefaction. This fall of the density behind the shock could be an alternative explanation for the dimming of EUV observations (see also Fig. 3), which are usually associated with the eruptive volume expansion of CMEs (Chen et al. 2002). At the chromospheric level, we note a deep persistent vertical compression (first step: $x \leq 170 \mathrm{Mm}$ ) that pushes down the interface chromosphere-corona, and a travelling front of density enhancement (second step: $170 \mathrm{Mm} \leq x \leq 220 \mathrm{Mm}$ ) more diffuse and less intense than the coronal one. Taking into account that the $\mathrm{H} \alpha$ opacity in the upper chromosphere is mainly sensitive to the mass density (the upper chromosphere can be considered as an optically thin media) and only weakly sensitive to the temperature (Leenaarts et al. 2012), we assume that this density profile gives account of the Moreton disturbance. As in White et al. (2014) (see fig. 3 of their paper), we note that there is a lag between the chromospheric perturbation and the coronal signal. In this case, at $t=300 \mathrm{~s}$, the chromospheric perturbation lags $\approx 30 \mathrm{Mm}$ behind the coronal signal. This is reinforced by the observation of the early activation of two distant filaments with respect to the chromospheric Moreton wave evolution, even in regions where it is no longer detectable, (Francile et al. 2013). If we consider different times (not shown in the figure), after the initial transitory, we find that the two signals travel with similar speeds and trajectories. In Fig. 6 we present the distance travelled by a chromospheric disturbance as a function of time for different uniform magnetic field values. A typical Moreton wave kinematics is obtained (see Table 1), i.e. a strong initial deceleration, that gradually diminishes which is larger for larger magnetosonic velocities (stronger magnetic fields). This characteristic behaviour is in agreement with the results in Francile et al. (2013) where we detected a Moreton wave with an initial deceleration of $\sim-30 \mathrm{~km} \mathrm{~s}^{-1}$ that diminishes to $\sim-2.4 \mathrm{~km} \mathrm{~s}^{-1}$ in an almost quadratic trend before it finishes in a linear one. The $t$ values of the table are the first detection times of the chromospheric travelling perturbation. The initial time interval with a lack of data is the time that takes the vertical coronal front, travelling with a fast

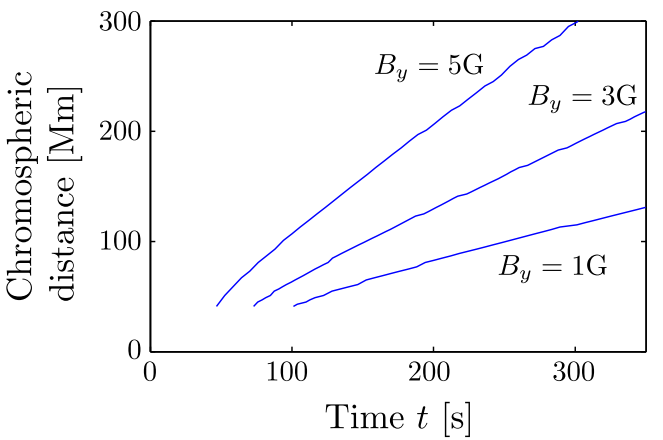

Figure 6. Distances travelled by the Moreton wave as a function of time, for different values of the magnetic field. $\Delta p / p=100$.

Table 1. Simulated models: $B_{0}$ is the initial background magnetic field, $v$ is the velocity in $x$ direction, $a$ is the acceleration and $t$ is the time of the first disturbance detection.

\begin{tabular}{lccc}
\hline$B_{0}[\mathrm{G}]$ & $v\left[\mathrm{~km} \mathrm{~s}^{-1}\right]$ & $a\left[\mathrm{~km} \mathrm{~s}^{-2}\right]$ & $t[\mathrm{~s}]$ \\
\hline 1 & 594 & -7 & 100 \\
3 & 1065 & -13 & 74 \\
5 & 1876 & -35 & 46 \\
\hline
\end{tabular}
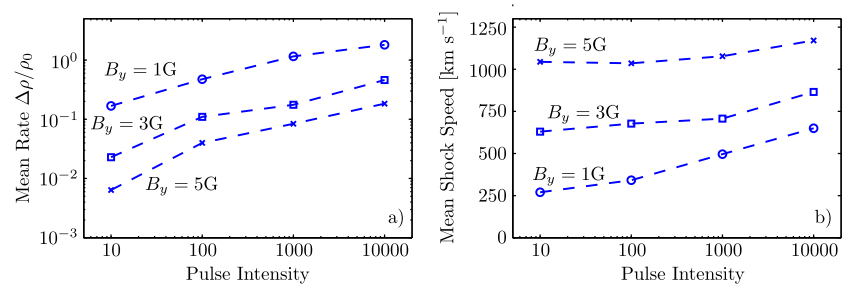

Figure 7. Coronal mean compression ratio (a) and velocity (b) for the shock wave considering different values of the magnetic field strength and the pressure pulse intensity.

magnetosonic shock speed, to arrive to the corona-chromosphere interface. However, the Moreton wave detection requires that the coronal perturbation compresses the chromosphere beyond a certain threshold, which was not taken into account to perform the figure. As in the observations, the simulations show that the chromospheric wave speed gradually becomes slower until the shock evolves to an ordinary fast magnetosonic disturbance (the linear region of the curves in Fig. 1 and Fig. 6) (Warmuth et al. 2004b). In Francile et al. (2013) a compression ratio of $\Delta \rho \gtrsim 0.08 \times \rho_{0}$ was required to obtain a detectable chromospheric perturbation which leads to a certain distance between the radiant point (the probable location of the single wave source projected into the chromosphere) and the place where the Moreton wave is initially visible. Fig. 7 shows the temporal average of both, (a) the compression ratio $\left(\Delta \rho / \rho_{0}\right)$ and (b) the front velocity of the coronal shock as a function of the pressure pulse strength for different magnetic field values. As mentioned before, the velocity and the compression ratio are mainly defined by the magnetic field intensity: strong fields $(\beta \ll 1)$ cause large shock speeds but small compression ratios. Moreover, the coronal shock speed is almost constant while varying the pressure pulse, specially for large values of the magnetic field, e.g. an increase of three orders of magnitude in $\Delta p / p$ implies an enhancement of the shock speed of $\sim 130$ per cent for a magnetic field of $B_{0}=1 \mathrm{G}$ and, of $\sim 10$ per cent for $B_{0}=5 \mathrm{G}$. Note that to obtain a linear shock speed trend of about 


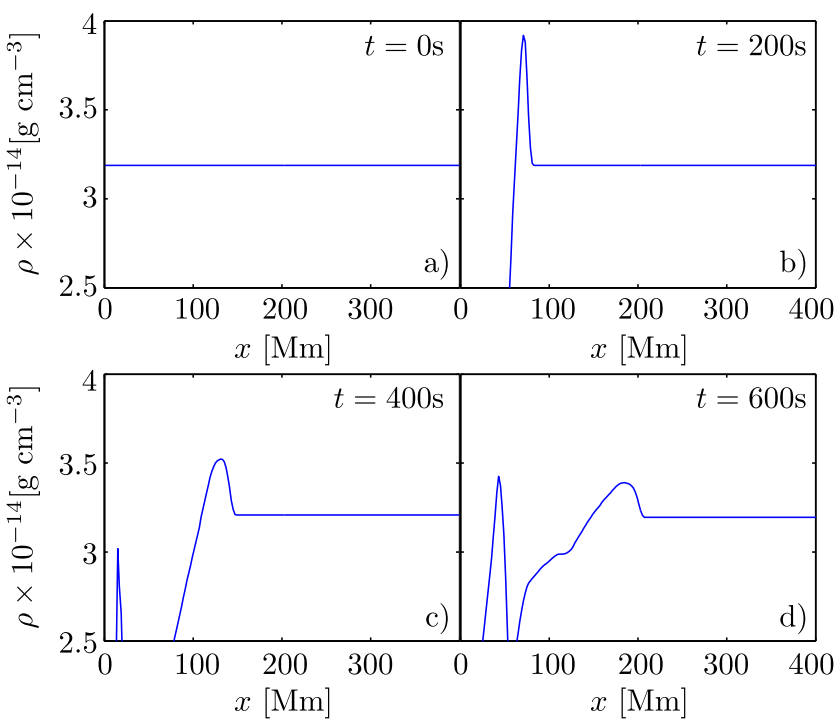

Figure 8. Density profiles at the chromospheric surface for different times showing the evolution of the Moreton wave. The magnetic field strength is $B_{0}=1 \mathrm{G}$ and the pressure pulse is 50 times larger than the ambient pressure.

$700 \mathrm{~km} \mathrm{~s}^{-1}$ (as in Fig. 1, which is also the typical averaged Moreton wave speed; Zhang et al. 2011), we need a magnetic field $\geq 3 \mathrm{G}$, but due to the rapid decrease of the compression ratio with increasing magnetic fields, it could be possible that the corresponding coronal compression ratio is insufficient to produce a detectable chromospheric compression with the HASTA instruments (a compression ratio of 8 per cent is required), even for the larger allowed values of the pressure pulse. This would be in accordance with the fact that, when detected, the Moreton events are generally associated with intense flares with an impulsive phase, usually leading to type II radio burst (Uchida 1968). To analyse if the coronal shock is capable to produce a detectable Moreton wave we study the chromospheric density profiles. We plot the density values at the $y$-coordinate positions lying in a slice (along the $x$-coordinate) located just below the unperturbed upper chromospheric layer as depicted in Fig. 8. Time $t=0 \mathrm{~s}$ (Fig. 8a) corresponds to the flare ignition and subsequent times indicate the evolution of the Moreton wave (see the chromospheric density profiles given in Figs 8(b)-(d), for $t=200$, $400,600 \mathrm{~s}$, respectively). The expected morphology of the wave is reproduced, i.e. an increasing width of the front and a decreasing amplitude of the wave with increasing time/distance. The fall in the density values behind the wave is related to the way the measurement is performed. As the density profile is obtained considering a slice just below the unperturbed interface corona-chromosphere, the coronal shock compresses the chromosphere from above enhancing the density (see Fig. 8b) and the top of the chromosphere is pushed downwards. Hence, what is measured behind the wave is the density in the rarefaction region of the coronal shock, and not the density of the chromosphere. Thus, in order to analyse the chromospheric disturbance we evaluate the density profile considering an 'adaptive' interface corona-chromosphere. This is, we adjust the measurement to the downward movement of the chromospheric surface. Also, instead of simply taking the density values considering a single layer of computational cells, just below the interface, we construct a profile using the average density over a vertical region below the chromospheric surface. As the $\mathrm{H} \alpha$ core emission measure in the upper chromosphere is mainly sensitive to the density disturbance (Leenaarts et al. 2012), this method is better adjusted to the

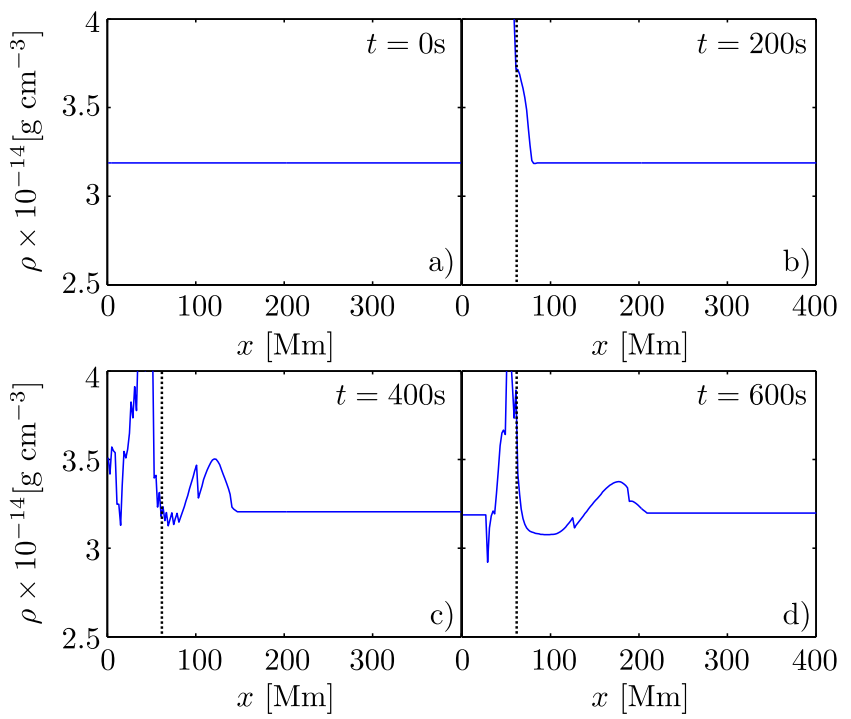

Figure 9. As Fig. 8, considering an adaptive chromospheric surface and an average density for a vertical distance of $d=1 \mathrm{Mm}$. The magnetic field strength is $B_{0}=1 \mathrm{G}$ and the pressure pulse is 50 times larger than the ambient pressure.

observations and allows a more precise comparison. In Figs 9(a)(d) we show the density profiles for the same experiment as in Figs 8(a)-(d), considering a chromospheric vertical region of $d=1$ $\mathrm{Mm}$ (measured downwards from the upper layer), for the average density calculation. Note, the qualitative agreement with the observational profiles consisting on a leading density enhancement (the Moreton wave) followed by an irregular trend (Balasubramaniam et al. 2010) (the 'activated' region behind the front, corresponding to the vertical shock compression over the chromosphere, see also the $\mathrm{H} \alpha$ line centre in fig. 3 of White et al. 2014). The differentiated two step behaviour can be appreciated from the figure. The vertical line separates the static region from the other one where the Moreton travelling perturbation can be appreciated.

\subsection{The temporal piston}

As noted before, the coronal shock speed is mainly determined by the magnetic field and almost independent of the pressure pulse intensity and the compression ratio (see Figs $7 \mathrm{a}-\mathrm{b}$ ). The speeds of the chromospheric perturbations will also be mostly determined by the magnetic field as they are supposed to be the consequence of the coronal shock sweeping over the chromospheric surface. However, a requirement for the perturbations to be observed is that the chromosphere is compressed beyond an instrumental threshold. Thus, it could happen that a very strong instantaneous pulse, not consistent with the plasma parameters, is required to produce a detectable perturbation. An alternative is to assume that the Moreton wave is triggered by a less intense pulse acting for a short time, i.e. a temporal piston.

As in Figs 7(a)-(b), in Figs 10(a)-(b) we show the coronal mean compression ratio and the mean shock speed, now as a function of the pulse duration. Note from Fig. 10(b), that the mean shock speed is also approximately independent of the pulse duration, i.e. is fundamentally determined by the magnetic field value. The speed initial values of Fig. 10(b) (at $t=0 \mathrm{~s}$ ) are the same as the ones of Fig. 7(b) for an instantaneous pulse intensity of 1000. Also, varying the duration of the pulse, from its instantaneous value to another of $50 \mathrm{~s}$ duration the compression rate increases up to $\sim 100$ per cent. 

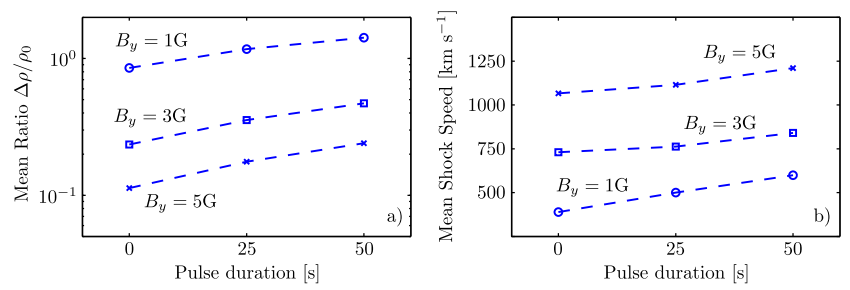

Figure 10. As Fig. 7, coronal mean compression ratio (a) and velocity (b) for the shock wave considering different values of the magnetic field strength as a function of the pulse duration with $\Delta p / p=1000$.

\subsection{The 2006 December 6 event}

We now consider the Moreton wave event on 2006 December 6. The aim of this analysis is to reproduce the observational curve obtained in Francile et al. (2013) for the time-distance relation displayed in Fig. 1. As seen previously, the main parameter required to determine the coronal shock speed is the magnetic field strength. To estimate this value we assume that the Moreton wave is due to a freely propagating coronal shock, which gradually decays to an ordinary fast-mode wave (Warmuth et al. 2001, 2004b). Considering the observational power-law curve $D$ of the Moreton wave given by Fig. 1, with the wave velocity corresponding to the later linear trend (at $t \geq 18: 49 \mathrm{UT}$ ) we can calculate the required magnetic field strength of $B_{0} \approx 3.53 \mathrm{G}$ for a fast magnetosonic speed $v_{\mathrm{fm}} \approx 720 \mathrm{~km} \mathrm{~s}^{-1}$. A distance of $35 \mathrm{Mm}$ - between the pressure pulse location and the chromospheric surface - is required to reproduce the phenomenological delay time between the flare ignition and the emergence of the chromospheric disturbance, i.e. $\sim 100 \mathrm{~s}$. We assumed a coronal temperature of $T=1.6 \mathrm{MK}$ and a coronal number density of $n=1.2 \times 10^{8} \mathrm{~cm}^{-3}$. The pressure pulse intensity is a crucial parameter in the simulation since it needs to be able to compensate the effect of the large magnetic field (associated with lower compression ratio values, see Fig. 7a) and produce a sufficiently strong compression wave in the chromosphere to be detected by the HASTAtelescope. The measurement of the detectable perturbation is performed by analysing if the density perturbation over the interface corona-chromosphere is larger than the threshold value starting from the larger distances towards the smaller ones for each time. Considering these requirements we first used the maximum admissible temperature and density values to set the pressure inside the flaring region which corresponded to $\Delta p / p \approx 10000$, the larger values in Fig. 7 .

Fig. 11 (blue circles) shows the obtained Moreton wave trajectory for $B_{0}=3.53 \mathrm{G}$. The dashed line corresponds to the observational data reported in Francile et al. (2013) and the circles indicate the Moreton wavefront obtained in our simulation. We assume that radiative losses can be treated supposing that the upper chromospheric region is an optically thin media (see e.g. Gayley \& Canfield 1991). Thus, we considered that the emission measure is proportional to the square of the particle density. The data indicating a Moreton front are the perturbations beyond the threshold. They are obtained measuring the data from the larger to the smaller distances. The final detection of the Moreton perturbation occurs when the intensity has weakened below the threshold $(t \sim 450 \mathrm{~s})$. The circles corresponding to later times in Fig. 11, $(\sim 420 \mathrm{~s}<t<\sim 500 \mathrm{~s})$ represent the stationary depression produced by the persistent vertical coronal compression (first step). These features were reported as persistent static brightenings and correspond to chromospheric $\mathrm{H} \alpha$ features (Delannée, Hochedez \& Aulanier 2007; White et al. 2014). In Francile et al. (2013) we find that the Moreton wave is

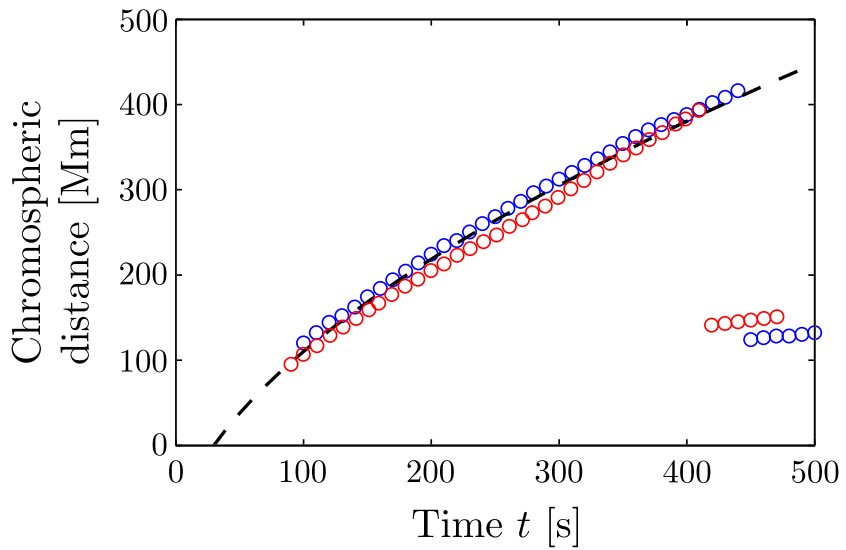

Figure 11. Comparison between the observational curve and the numerical simulation for $B_{0}=3.53 \mathrm{G}$. The dashed line represents the $2 \mathrm{D}$ averaged chromospheric distance travelled by the Moreton wave registered on 2006 December 6 by Francile et al. (2013). The blue and red circles indicate the travelling wavefront obtained by the simulation applying an instantaneous pressure pulse of $\Delta p / p \approx 10000$ and a temporal pressure pulse of $\Delta p / p \approx 1000$, applied during $40 \mathrm{~s}$, respectively. A threshold of 8 per cent in the compression ratio of an optically thin media is considered.

detected $130 \mathrm{Mm}$ away from the radiant point. Accordingly, in our simulations, this distance $(\sim 120 \mathrm{Mm})$ corresponds to a peak of the perturbation. Considering the evolution at different times we obtain, as in White et al. (2014), that the chromospheric perturbation of 2006 December, displays a characteristic down-up vertical velocity pattern that lags $\approx 50 \mathrm{Mm}$ behind the coronal signal and travels with almost the same speed and trajectory as the coronal one (see Fig. 5). Large values of pressure pulses can be expected coming from super-Alfvénic reconnection outflows as mentioned by Mann \& Warmuth (2011). However, less impulsive phenomenon could be accomplished providing the energy to generate almost the same chromospheric perturbation if a less impulsive event lasts a larger time. The red circles in Fig. 11 correspond to the less intense temporal piston case that adjusts the observational curve: $\Delta p / p \approx 1000$, applied during $40 \mathrm{~s}$ which could resemble the action of the expanding flanks of a CME (Temmer et al. 2009). This pulse duration corresponds to the minimum value that produces a compression ratio beyond the instrumental threshold.

\section{CONCLUSIONS}

Fig. 11 shows that the kinematics of the Moreton event of 2006 December 6 can be reproduced assuming a blast-wave scenario, originated from a single source, that produces a coronal fast mode MHD shock that sweeps the chromosphere generating the Moreton perturbation. This source could be generated by a flare that ignites the blast-wave or by the expansion of CME flanks due to the flare ignition. We found that, using typical coronal and chromospheric parameters, a wave of large amplitude that propagates decelerating (starting with an initial $a_{0} \geq-30 \mathrm{~km} \mathrm{~s}^{-2}$ ) with a decreasing amplitude until it acquires a final fast magnetosonic free speed linear trend of $v_{\mathrm{fm}} \approx 720 \mathrm{~km} \mathrm{~s}^{-1}$ is obtained. As in White et al. (2014) and in Francile et al. (2013) we found that the chromospheric perturbation displays a characteristic down-up vertical velocity pattern that lags behind the coronal signal and travels with the same speed and trajectory as the coronal one (see Fig. 5). A uniform magnetic field value of $B_{0} \approx 3.53 \mathrm{G}$ and a distance of $35 \mathrm{Mm}$, between the pressure pulse location and the corona-chromospheric interface, are required 
to reproduce the phenomenological delay time between the flare ignition and the emergence of the chromospheric disturbance. The first detection of the Moreton wavefront is $\approx 120 \mathrm{Mm}$ far from the radiant point which is consistent with the value obtained in Francile et al. (2013).

It has been argued that the flare explosion alone is not enough to give account of the Moreton event due to the discrepancy between the frequency of flare phenomenon and the relatively rare observations of coronal shocks. Thus, another mechanism would be necessary or a very special condition must be accomplished to give account of the shock formation that produces the large-scale Moreton event. If the modelling used here is an accurate one, our results show that, given typical coronal and chromospheric parameters, a set of limiting conditions are required to obtain both, a final linear trend associated with a fast magnetosonic speed and a strong pressure pulse able to generate a detectable compression ratio. The final fast magnetosonic free speed requires a definite magnetic field intensity (Fig. 7b), but if the intensity of the magnetic field is larger the compression ratio values are small and hinder the wave detection (Fig. 7a). Accordingly, as seen in the appendix, the flare expansion should be located at the periphery of the AR in order to avoid large magnetic field values (where $\beta \sim 1 \times 10^{-4}$ ). Also, the fact that a very strong pressure pulse (limited by the admissible values of temperature and density) was required to obtain an emission enhancement of only 8 percent for later times reinforces the argument.

\section{ACKNOWLEDGEMENT}

This work was supported by the grant PIP-CONICET 20080102773 .

\section{REFERENCES}

Aschwanden M. J., 2005, Physics of the Solar Corona. An Introduction with Problems and Solutions, 2nd edn. Praxis Publishing Ltd, Chichester Athay R. G., Moreton G. E., 1961, ApJ, 133, 935

Aurass H., Shibasaki K., Reiner M., Karlický M., 2002, ApJ, 567, 610

Balasubramaniam K. S. et al., 2010, ApJ, 723, 587

Cécere M., Schneiter M., Costa A., Elaskar S., Maglione S., 2012, ApJ, 759, 79

Chen P. F., Shibata K., 2000, ApJ, 545, 524

Chen P. F., Wu S. T., Shibata K., Fang C., 2002, ApJ, 572, L99

Chen P. F., Ding M. D., Fang C., 2005, Space Sci. Rev., 121, 201

Cliver E. W., Webb D. F., Howard R. A., 1999, Sol. Phys., 187, 89

Costa A., Elaskar S., Fernández C. A., Martínez G., 2009, MNRAS, 400, L85

Delaboudinière J.-P. et al., 1995, Sol. Phys., 162, 291

Delannée C., Hochedez J.-F., Aulanier G., 2007, A\&A, 465, 603

Draine B. T., 2011, Physics of the Interstellar and Intergalactic Medium. Princeton Univ. Press, Princeton, NJ

Fernández C. A., Costa A., Elaskar S., Schulz W., 2009, MNRAS, 400, 1821

Francile C., Costa A., Luoni M. L., Elaskar S., 2013, A\&A, 552, A3

Fryxell B. et al., 2000, ApJS, 131, 273

Gayley K. G., Canfield R. C., 1991, ApJ, 380, 660

Gilbert H. R., Holzer T. E., 2004, ApJ, 610, 572

Gilbert H. R., Daou A. G., Young D., Tripathi D., Alexander D., 2008, ApJ, 685,629

Guo L.-J., Bhattacharjee A., Huang Y.-M., 2013, ApJ, 771, L14

Handy B. N. et al., 1999, Sol. Phys., 187, 229

Knock S. A., Cairns I. H., Robinson P. A., Kuncic Z., 2001, J. Geophys. Res., 106, 25041

Lee D., Deane A. E., Federrath C., 2009, in Pogorelov N. V., Audit E., Colella P., Zank G. P., eds, ASP Conf. Ser. Vol. 406, Numerical Modelling of Space Plasma Flows: ASTRONUM-2008, Astron. Soc. Pac., San Francisco, p. 243

Leenaarts J., Carlsson M., Hansteen V., Rutten R. J., 2007, A\&A, 473, 625 Leenaarts J., Carlsson M., Rouppe van der Voort L., 2012, ApJ, 749, 136

Magdalenić J., Marqué C., Zhukov A. N., Vršnak B., Veronig A., 2012, ApJ, 746,152

Mann G., Warmuth A., 2011, A\&A, 528, A104

Mei Z., Shen C., Wu N., Lin J., Murphy N. A., Roussev I. I., 2012, MNRAS, 425,2824

Moreton G. E., 1960, AJ, 65, 494

Moreton G. E., Ramsey H. E., 1960, PASP, 72, 357

Onofri M., Primavera L., Malara F., Veltri P., 2004, Phys. Plasmas, 11, 4837

Su W., Cheng X., Ding M. D., Chen P. F., Sun J. Q., 2015, ApJ, 804, 88

Temmer M., Vršnak B., Žic T., Veronig A. M., 2009, ApJ, 702, 1343

Thompson B. J., Plunkett S. P., Gurman J. B., Newmark J. S., St. Cyr O. C., Michels D. J., 1998, Geophys. Res. Lett., 25, 2465

Torrilhon M., 2003, J. Plasma Phys., 69, 253

Uchida Y., 1968, Sol. Phys., 4, 30

Uchida Y., Altschuler M. D., Newkirk G., Jr, 1973, Sol. Phys., 28, 495

Vršnak B., Cliver E. W., 2008, Sol. Phys., 253, 215

Vršnak B., Lulić S., 2000a, Sol. Phys., 196, 157

Vršnak B., Lulić S., 2000b, Sol. Phys., 196, 181

Vršnak B., Warmuth A., Brajša R., Hanslmeier A., 2002a, A\&A, 394, 299

Vršnak B., Magdalenić J., Aurass H., Mann G., 2002b, A\&A, 396, 673

Warmuth A., Vršnak B., Aurass H., Hanslmeier A., 2001, ApJ, 560, L105

Warmuth A., Vršnak B., Magdalenić J., Hanslmeier A., Otruba W., 2004a, A\&A, 418, 1101

Warmuth A., Vršnak B., Magdalenić J., Hanslmeier A., Otruba W., 2004b, A\&A, 418, 1117

Warmuth A., Mann G., Aurass H., 2005, ApJ, 626, L121

White S., Balasubramaniam K., Cliver E., 2014, AFRL-RV-PS-TP-20140004. Air Force Research Laboratory, Preprint - Technical Paper, p. 22

Wu S. T., Zheng H., Wang S., Thompson B. J., Plunkett S. P., Zhao X. P., Dryer M., 2001, J. Geophys. Res., 106, 25089

Zhang Y., Kitai R., Narukage N., Matsumoto T., Ueno S., Shibata K., Wang J., 2011, PASJ, 63, 685

Zhukov A. N., 2011, J. Atmos. Sol.-Terr. Phys., 73, 1096

Zhukov A. N., Auchère F., 2004, A\&A, 427, 705

\section{APPENDIX: IMPORTANCE OF THE $\beta$ PARAMETER TO IGNITE A CORONAL SHOCK}

Consider a steady shock plane-parallel to the magnetic field $\left(B_{0}\right)$ and normal to the $x$-coordinate. Making use of the Rankine-Hugoniot conditions, the conservation laws for mass, momentum, energy and magnetic flux read (see e.g. Torrilhon 2003 and Draine 2011):

$$
\begin{aligned}
& \rho_{0} v_{0}=\rho_{1} v_{1}, \\
& v_{0} B_{0}=v_{1} B_{1}, \\
& \rho_{0} v_{0}^{2}+p_{0}+\frac{B_{0}^{2}}{8 \pi}=\rho_{1} v_{1}^{2}+p_{1}+\frac{B_{1}^{2}}{8 \pi}, \\
& v_{0}\left[\frac{1}{2} \rho_{0} v_{0}^{2}+\frac{\gamma}{\gamma-1} v_{0} p_{0}+v_{0} \frac{B_{0}^{2}}{8 \pi}\right] \\
& =v_{1}\left[\frac{1}{2} \rho_{1} v_{1}^{2}+\frac{\gamma}{\gamma-1} v_{1} p_{1}+v_{1} \frac{B_{1}^{2}}{8 \pi}\right],
\end{aligned}
$$

where the subscripts 0 and 1 denote pre-shock and post-shock conditions, respectively, $v$ is the velocity in $x$-direction in a frame fixed to the shock front.

The non-trivial solution is one that produces different values on each side of the discontinuity. Considering the pre-shock plasma at rest, it results that $v_{0}=v_{s}$ in the moving frame, where $v_{s}$ is the 
shock speed. Defining the compression ratio $X=\rho_{1} / \rho_{0}$ (Draine 2011):

$2(2-\gamma) X^{2}+\gamma\left[(\gamma-1) \beta M_{\mathrm{s}}^{2}+2(1+\beta)\right] X$

$$
-\gamma(\gamma+1) \beta M_{\mathrm{s}}^{2}=0
$$

$M_{\mathrm{s}}=v_{\mathrm{s}} / c_{\mathrm{s}}$ is the shock Mach number.

As the shock speed is equal or larger than the fast magnetosonic speed $v_{\mathrm{fm}}=\left(v_{A}^{2}+c_{\mathrm{s}}^{2}\right)^{1 / 2}$, a shock compression implies $v_{s}=\alpha v_{\mathrm{fm}}$, where $\alpha \geq 1$, thus:

$M_{\mathrm{s}}^{2}=\alpha^{2} \frac{v_{\mathrm{A}}^{2}+c_{\mathrm{s}}^{2}}{c_{\mathrm{s}}^{2}}=\alpha^{2}\left[\frac{2}{\gamma \beta}+1\right]$.

We can rewrite equation (A2) in terms of the parameter $\beta$

$$
\begin{aligned}
& 2(2-\gamma) X^{2}+\left[\alpha^{2}(\gamma-1)(2+\gamma \beta)+2(1+\beta)\right] X \\
& -\alpha^{2}(\gamma+1)(2+\gamma \beta)=0 .
\end{aligned}
$$

Note that, in the case of interest $(1 \leq \gamma \leq 2)$, equation (A4) has only one positive root which satisfies $X \geq 1$. The limit $X \rightarrow 1$, is obtained when $\beta \rightarrow 0$, independently of the values of $\gamma$ and $\alpha$. Thus, the flare-associated pressure pulse cannot ignite a shock wave in strong field regions, where $\beta \rightarrow 0$. A detailed study on MHD shock wave formation can be found in Vršnak \& Lulić (2000a) and Vršnak \& Lulić (2000b).

This paper has been typeset from a $\mathrm{T}_{\mathrm{E}} \mathrm{X} / \mathrm{L} \mathrm{T} \mathrm{E} \mathrm{X}$ file prepared by the author. 